Fernando José Spanhol

Giovani Mendonça Lunardi

Márcio Vieira de Souza

(organizadores)

\title{
Tecnologias da informação e comunicação na segurança pública e direitos humanos
}

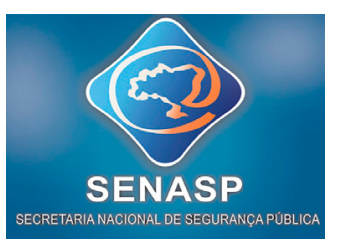


Tecnologias da informação e comunicação na segurança pública e direitos humanos

๑ 2016 Fernando José Spanhol / Giovani Mendonça Lunardi / Márcio Vieira de Souza (organizadores)

Editora Edgard Blücher Ltda.

\section{Blucher}

Rua Pedroso Alvarenga, $1245,4^{\circ}$ andar

04531-934 - São Paulo - SP - Brasil

Tel 5511 3078-5366

contato@blucher.com.br

www.blucher.com.br

Segundo Novo Acordo Ortográfico, conforme 5.ed. do Vocabulário Ortográfico da Língua Portuguesa, Academia Brasileira de Letras, março de 2009.

É proibida a reprodução total ou parcial por quaisquer meios, sem autorização escrita da Editora.

Todos os direitos reservados pela Editora Edgard Blücher Ltda.

\section{FICHA CATALOGRÁFICA}

Tecnologias da informação e comunicação na segurança pública e direitos humanos / organização de Fernando José Spanhol, Giovani Mendonça Lunardi, Márcio Vieira de Souza. - São Paulo: Blucher, 2016.

206 p.; pdf.

\section{Bibliografia}

ISBN 978-85-8039-176-3 (e-book)

ISBN 978-85-8039-177-0 (impresso)

Open Access

1. Tecnologia da informação 2. Segurança pública 3. Direitos humanos I. Spanhol, Fernando José II. Lunardi, Giovani Mendonça III. Souza, Márcio Vieira de

16-0587

CDD 004
Índice para catálogo sistemático:

1. Tecnologia da informação 


\section{Agradecimentos}

Esta obra foi elaborada com recursos da Secretaria Nacional de Segurança Pública (SENASP), do Ministério da Justiça (MJ), por meio do Edital SENASP $\mathrm{n}^{\circ}$ 006/2012, vinculado à Rede Nacional de Altos Estudos em Segurança Pública (RENAESP).

Os temas aqui apresentados são frutos de pesquisas executadas por professores e alunos do curso de especialização em Tecnologias da Informação e Comunicação Aplicadas à Segurança Pública e Direitos Humanos (turma 2013-2015), da Universidade Federal de Santa Catarina - Campus Araranguá, pertencente ao Programa de Pós-Graduação do Mestrado Interdisciplinar em Tecnologias da Informação e Comunicação (PPGTIC).

A organização desta obra coube aos pesquisadores do Laboratório de Mídia e Conhecimento do Campus Araranguá (UFSC/SC). 


\section{Conselho editorial da coleção "mídia, educação, inovação e conhecimento"}

COORDENADOR DO CONSELHO EDITORIAL

Dr. Marcio Vieira de Souza - Universidade Federal de Santa Catarina (UFSC)

CONSELHO EDITORIAL

Dr. Alexandre Marino Costa - Universidade Federal de Santa Catarina (UFSC)

Dr. ${ }^{a}$ Alexandra Okada - Open University (Inglaterra)

Dr. ${ }^{a}$ Araci Hack Catapan - Universidade Federal de Santa Catarina (UFSC)

Dr. Carlos Alberto de Souza - Universidade Estadual de Ponta Grossa (UEPG)

Dr. ${ }^{a}$ Clarissa Stefani - Universidade Federal de Santa Catarina (UFSC)

Dr. ${ }^{a}$ Eliane Schlemmer - Universidade do Vale do Rio dos Sinos (Unisinos)

Dr. Francisco Antonio Pereira Fialho - Universidade Federal de Santa Catarina (UFSC)

Dr. Pablo Lucas - University College Dublin (Irlanda)

Dr. Fernando Jose Spanhol - Universidade Federal de Santa Catarina (UFSC)

Dr. Giovani Mendonça Lunardi - Universidade Federal de Santa Catarina (UFSC)

Dr. ${ }^{a}$ Greicy K. Spanhol Lenzi - Faculdade AEROTD (Faero)

Dr. José Manuel Moran - Universidade de São Paulo (USP)

Dr. Kamil Giglio - Teltec Solutions/Faculdade AEROTD (Faero)

Dr. ${ }^{a}$ Karina Marcon - Universidade do Estado de Santa Catarina (Udesc)

Dr. Luiz Palazzo - Universidade Católica de Pelotas (UCPEL)

Dr. Marcio Vieira de Souza - Universidade Federal de Santa Catarina (UFSC)

Dr. ${ }^{a}$ Mara Lucia Fernandes Carneiro - Universidade Federal do Rio Grande do Sul (UFRGS)

Dr. ${ }^{a}$ Margarita Victoria Gomez - Universidade Federal do Rio de Janeiro (UFRJ)

Dr. ${ }^{a}$ Marina Keiko Nakayama - Universidade Federal de Santa Catarina (UFSC)

Dr. ${ }^{a}$ Ofelia Morales - Universidade Estadual de Ponta Grossa (UEPG)

Dr. ${ }^{a}$ Patricia Lupion Torres - Pontifícia Universidade Católica do Paraná (PUC-PR)

Dr. ${ }^{a}$ Patricia Jantsch Fiuza - Universidade Federal de Santa Catarina (UFSC)

Dr. ${ }^{a}$ Ricardo Azambuja Silveira - Universidade Federal de Santa Catarina (UFSC)

Dr. Robson Rodrigues Lemos - Universidade Federal de Santa Catarina (UFSC)

Dr. ${ }^{a}$ Roselaine Ripa - Universidade do Estado de Santa Catarina (Udesc)

Dr. ${ }^{a}$ Silvia Quevedo - Universidade Federal de Santa Catarina (UFSC)

Dr. Tarcisio Vanzin - Universidade Federal de Santa Catarina (UFSC)

Dr. ${ }^{a}$ Vânia Ribas Ulbricht - Universidade Federal de Santa Catarina (UFSC) 


\section{Sobre os autores}

Alexandre Leopoldo Gonçalves - Doutor em Engenharia de Produção pela Universidade Federal de Santa Catarina (UFSC). Atualmente, é professor adjunto e professor permanente dos Programas de Pós-Graduação em Engenharia e Gestão do Conhecimento (PPGEGC) e em Tecnologias da Informação e Comunicação (PPGTIC), todos na UFSC.

Alberto Felipe Friderichs Barros - Mestrando do Programa de Pós-Graduação em Tecnologias da Informação e Comunicação pela Universidade Federal de Santa Catarina (PPGTIC/UFSC). Graduado em Tecnologia em Telecomunicações pela Universidade do Extremo Sul Catarinense. Atualmente, é coordenador de Tecnologia da Informação e Comunicação no Instituto Federal de Santa Catarina (IFSC) - Campus Criciúma.

Daiane Oliveira João - Especialista em Tecnologias da Informação e Comunicação Aplicadas à Segurança Pública e Direitos Humanos pela Universidade Federal de Santa Catarina (Pós TIC Senasp). Bacharel em Ciência da Computação pela Universidade do Extremo Sul de Santa Catarina. Atualmente, é analista de sistemas.

Eduardo Santos Acosta - Especialista em Tecnologias da Informação e Comunicação Aplicadas à Segurança Pública e Direitos Humanos pela Universidade Federal de Santa Catarina (Pós TIC Senasp). Bacharel em Direito pela Universidade de Santa Cruz do Sul. Atualmente, é bombeiro militar do Rio Grande do Sul.

Elbio Carlos Bock - Especialista em Tecnologias da Informação e Comunicação Aplicadas à Segurança Pública e Direitos Humanos pela Universidade Federal de Santa Catarina (Pós TIC Senasp). Bacharel em Direito pela Universidade Luterana do Brasil. Atualmente, é agente da Polícia Civil do Rio Grande do Sul.

Eliane Pozzebon - Doutora em Engenharia Elétrica com ênfase em Automação e Sistemas pela Universidade Federal de Santa Catarina (UFSC). Atualmente, é 
professora adjunta e professora permanente do Programa de Pós-Graduação em Tecnologias da Informação e Comunicação (PPGTIC), ambos na UFSC.

Emerson Wendt - Mestre em Direito pelo Centro Universitário La Salle (UnilaSalle), de Canoas (RS). Graduado em Ciências Jurídicas e Sociais/Direito pela Universidade Federal de Santa Maria (UFSM). Atualmente, é chefe de Polícia na Polícia Civil do Rio Grande do Sul.

Fernanda Todesco Nunes - Especialista em Tecnologias da Informação e Comunicação Aplicadas à Segurança Pública e Direitos Humanos pela Universidade Federal de Santa Catarina (Pós TIC Senasp). Licenciada em Matemática pela UFSC. Atualmente, é agente da Polícia Civil de Santa Catarina.

Fernando Henrique Borges Ferreira - Especialista em Tecnologias da Informação e Comunicação Aplicadas à Segurança Pública e Direitos Humanos pela Universidade Federal de Santa Catarina (Pós TIC Senasp). Bacharel em Direito pela UFSC. Atualmente, é escrivão da Polícia Civil.

Fernando José Spanhol - Doutor em Mídia e Conhecimento pela Universidade Federal de Santa Catarina (UFSC). Atualmente, é professor adjunto e professor permanente dos Programas de Pós-Graduação em Engenharia e Gestão do Conhecimento (PPGEGC) e em Tecnologias da Informação e Comunicação (PPGTIC), todos na UFSC.

Giovani Mendonça Lunardi - Pós-doutor em Filosofia (PPG Filosofia) pela Unisinos junto à Cátedra Unesco/Unisinos de Direitos Humanos e ao Grupo de Pesquisa Ética, Biopolítica e Alteridade. Atua como professor adjunto e professor permanente dos Programas de Pós-Graduação em Tecnologias da Informação e Comunicação (PPGTIC) e em Energia e Sustentabilidade (PPGES), todos na UFSC.

Herbson de Carvalho - Especialista em Tecnologias da Informação e Comunicação Aplicadas à Segurança Pública e Direitos Humanos pela Universidade Federal de Santa Catarina (Pós TIC Senasp). Bacharel em Administração pela Universidade Norte do Paraná. Atualmente, é soldado qpm-1 - Brigada Militar do Rio Grande do Sul.

João Bosco da Mota Alves - Doutor em Engenharia Elétrica pela coordenação dos cursos de Pós-Graduação em Engenharia da Universidade Federal do Rio de Janeiro (COPPE/UFRJ). Atualmente, é professor voluntário nos Programas de Pós-Graduação em Engenharia e Gestão do Conhecimento (PPGEGC) e em Tecnologias da Informação e Comunicação (PPGTI), ambos na UFSC.

Joel Souza de Oliveira - Mestrando em Tecnologias da Informação e Comunicação pela Universidade Federal de Santa Catarina (PPGTIC/UFSC). Especialista em Tecnologias da Informação e Comunicação Aplicadas à Segurança Pública e Direitos Humanos pela UFSC (Pós TIC Senasp). Bacharel em Ciên- 
cias Jurídicas e Sociais pela Pontifícia Universidade Católica do Rio Grande do Sul (PUC-RS). Atualmente, é delegado de Polícia da Polícia Civil do Estado do Rio Grande do Sul.

Josiel Pereira - Mestrando do Programa de Pós-Graduação em Tecnologias da Informação e Comunicação pela Universidade Federal de Santa Catarina (PPGTIC/UFSC). Bacharel em Tecnologias da Informação e Comunicação pela UFSC. Atualmente, é bolsista pela Fundação de Apoio à Pesquisa Científica e Tecnológica do Estado de Santa Catarina (Fapesc).

Juarez Bento da Silva - Doutor em Engenharia e Gestão do Conhecimento pela Universidade Federal de Santa Catarina (UFSC). Atua como professor adjunto e professor permanente do Programa de Pós-Graduação em Tecnologias da Informação e Comunicação (PPGTIC) na UFSC.

Laudelino Joaquim - Especialista em Tecnologias da Informação e Comunicação Aplicadas à Segurança Pública e Direitos Humanos pela Universidade Federal de Santa Catarina (Pós TIC Senasp). Bacharel em Ciência da Computação pela Universidade do Sul de Santa Catarina. Atualmente, é soldado da Polícia Militar de Santa Catarina.

Leandro da Silva dos Reis - Especialista em Tecnologias da Informação e Comunicação Aplicadas à Segurança Pública e Direitos Humanos pela Universidade Federal de Santa Catarina (Pós TIC Senasp). Bacharel em Direito pela Universidade do Sul de Santa Catarina. Atualmente, é agente da Polícia Civil de Santa Catarina.

Lorielcio da Silva Peres - Especialista em Tecnologias da Informação e Comunicação Aplicadas à Segurança Pública e Direitos Humanos pela Universidade Federal de Santa Catarina (Pós TIC Senasp). Bacharel em Administração pela UFSC. Atualmente, é gerente de projetos e consultor.

Luciana Bolan Frigo - Doutora em Engenharia Elétrica pela Universidade Federal de Santa Catarina (UFSC) e doutora em Informática pela Université de Toulouse 1 (2007). Atualmente, é professora adjunta e professora colaboradora do Programa de Pós-Graduação em Tecnologias da Informação e Comunicação (PPGTIC) na UFSC.

Márcio Giani Rosa de Matos - Especialista em Tecnologias da Informação e Comunicação Aplicadas à Segurança Pública e Direitos Humanos pela Universidade Federal de Santa Catarina (Pós TIC Senasp). Licenciado em História pela Universidade Luterana do Brasil. Atualmente, é sargento da Policia Militar de Santa Catarina.

Márcio Vieira de Souza - Doutor em Engenharia de Produção pela Universidade Federal de Santa Catarina (UFSC). É professor adjunto e professor permanente nos Programas de Pós-Graduação em Tecnologia da Informação e Comu- 
nicação (PPGTIC) e em Engenharia e Gestão do Conhecimento (PPGEGC), todos na UFSC.

Marta Adriana da Silva Cristiano - Doutoranda em Engenharia e Gestão do Conhecimento pela Universidade Federal de Santa Catarina (PPGEGC/UFSC). Graduada em Ciência da Computação pela Universidade do Sul de Santa Catarina. Atualmente, é pesquisadora do Laboratório de Experimentação Remota (RExLab) e do Grupo de Pesquisa INOVAmid - Subjetividade e Inovação em Mídias do Conhecimento na Sociedade em Rede (UFSC).

Natana Lopes Pereira - Bacharelanda em Tecnologias da Informação e Comunicação pela Universidade Federal de Santa Catarina (UFSC). Atualmente, é bolsista do curso Pós TIC Senasp - Especialização em Tecnologias da Informação e Comunicação Aplicadas à Segurança Pública e Direitos Humanos.

Norton Meidel Karam - Especialista em Tecnologias da Informação e Comunicação Aplicadas à Segurança Pública e Direitos Humanos pela Universidade Federal de Santa Catarina (Pós TIC Senasp). Graduado em Engenharia Química pela Pontifícia Universidade Católica do Rio Grande do Sul (PUC-RS). Atualmente, é perito criminal do Instituto Geral de Perícias do Rio Grande do Sul.

Paulo Cesar Leite Esteves - Doutor pelo Programa de Pós-Graduação do Departamento de Engenharia de Produção e Sistemas da Universidade Federal de Santa Catarina (UFSC). Atualmente é professor adjunto e professor permanente da Pós-Graduação em Tecnologias da Informação e Comunicação (PPGTIC), ambos na UFSC.

Priscila Cadorin Nicolete - Mestranda do Programa de Pós-Graduação em Tecnologias da Informação e Comunicação pela Universidade Federal de Santa Catarina (PPGTIC/UFSC). Bacharel em Tecnologias da Informação e Comunicação pela UFSC. Atualmente, é bolsista pelo Centro Anhanguera de Promoção e Educação Social (Capes).

Rangel Machado Simon - Mestrando do Programa de Pós-Graduação em Engenharia e Gestão do Conhecimento pela Universidade Federal de Santa Catarina (PPGEGC/UFSC). Bacharel em Tecnologias da Informação e Comunicação pela UFSC. Atualmente, é professor do Instituto Mix e pesquisador laboratório de mídia e informação da UFSC.

Roderval Marcelino - Doutor em Engenharia de Minas, Metalúrgica e de Materiais pela Universidade Federal do Rio Grande do Sul (UFRGS). Atua como professor adjunto e professor permanente do Programa de Pós-Graduação em Tecnologias da Informação e Comunicação (PPGTIC), ambos na UFSC.

Rosinei Freitas da Rosa - Especialista em Tecnologias da Informação e Comu- 
nicação Aplicadas à Segurança Pública e Direitos Humanos pela Universidade Federal de Santa Catarina (Pós TIC Senasp). Bacharel em Direito pela Universidade do Extremo Sul Catarinense. Atualmente, é soldado da Polícia Militar do Estado de Santa Catarina.

Solange Maria da Silva - Doutora em Engenharia de Produção e Sistemas pela Universidade Federal de Santa Catarina (UFSC). Atualmente, é professora adjunta na mesma instituição.

Sônia Maria Dall'Igna - Mestranda em Tecnologias da Informação e Comunicação pela Universidade Federal de Santa Catarina (PPGTIC/UFSC). Especialista em Tecnologias da Informação e Comunicação Aplicadas à Segurança Pública e Direitos Humanos pela Universidade Federal de Santa Catarina (Pós TIC Senasp). Bacharel em Ciências Jurídicas e Sociais pela Universidade do Vale do Rio dos Sinos. Atualmente, é diretora da Divisão de Prevenção e Educação (Dipe), do Departamento Estadual de Investigação do Narcotráfico da Polícia Civil do Rio Grande do Sul.

Thabata Clezar de Almeida - Mestranda pelo Programa de Pós-Graduação em Engenharia e Gestão do Conhecimento (PPGEGC/UFSC). Bacharel em Direito pela Universidade do Sul de Santa Catarina. Atualmente, é advogada da Ordem dos Advogados do Brasil de Santa Catarina (OAB-SC).

Vilson Gruber - Doutor em Engenharia de Minas, Metalúrgica e de Materiais pela Universidade Federal do Rio Grande do Sul (UFRGS). Atua como professor adjunto e professor permanente do Programa de Pós-Graduação em Tecnologias da Informação e Comunicação (PPGTIC), ambos na UFSC. 



\section{Sobre os organizadores}

Fernando José Spanhol - Doutor em Mídia e Conhecimento pela Universidade Federal de Santa Catarina (UFSC). Atualmente, é professor adjunto e professor permanente dos Programas de Pós-Graduação em Engenharia e Gestão do Conhecimento (PPGEGC) e em Tecnologias da Informação e Comunicação (PPGTIC), todos na UFSC.

Giovani Mendonça Lunardi - Pós-doutor em Filosofia (PPG Filosofia) pela Unisinos junto à Cátedra Unesco/Unisinos de Direitos Humanos e ao Grupo de Pesquisa Ética, Biopolítica e Alteridade. É professor adjunto e professor permanente dos Programas de Pós-Graduação em Tecnologias da Informação e Comunicação (PPGTIC) e em Energia e Sustentabilidade (PPGES), todos na UFSC.

Márcio Vieira de Souza - Doutor em Engenharia de Produção pela Universidade Federal de Santa Catarina (UFSC). É professor adjunto e professor permanente nos Programas de Pós-Graduação em Tecnologia da Informação e Comunicação (PPGTIC) e em Engenharia e Gestão do Conhecimento (PPGEGC), todos na UFSC. 



\section{Prefácio}

Muito me honra o convite para prefaciar um livro escrito por dezenas de mãos e mentes brilhantes e ávidas pelo debate acadêmico. Não poderia deixar de atender à convocação do Prof. Dr. Giovani M. Lunardi, da Universidade Federal de Santa Catarina (UFSC), Campus de Araranguá/SC, local onde tive o privilégio de palestrar.

Os autores inovam em seus escritos, abrangendo o uso da tecnologia dentro de um contexto - de Segurança Pública - em que o seu desuso já não pode ser vislumbrado, mas, por outro lado, não é privilegiado, incentivado ou, em outros termos, não tem a atenção que merece, seja pelos atores da segurança pública, seja pelos políticos que a usam como meio de promessa, principalmente se aliada ao contexto da atividade de inteligência. Aliás, os investimentos em tecnologias novas geralmente são colocados em segundo plano e as verdadeiras inovações e utilizações saem de aplicações parciais ou de iniciativas pessoais.

As políticas de segurança, embora tenham uma contemplação e diretriz nacional, nem sempre atendem às demandas regionais ou locais de cada Estado, principalmente quando estipulam apenas orientações teóricas e não vêm acompanhadas de incentivos financeiros, ou relativos a recursos materiais, ou em relação à formação dos profissionais de segurança pública. Os gestores contemporâneos têm, por isso, que se adequar às condições locais de produção de conhecimentos e, com base nas necessidades e demandas existentes, procurar atuar frente a uma conjuntura nacional e buscando, aliado às bases tecnológicas, a padronização de conceitos, estruturas e procedimentos relativos ao seu nicho de atuação, seja de polícia ostensiva, seja de polícia investigativa, seja de perícia, seja de atuação de defesa civil ou atuação em catástrofes naturais ou situações humanas provocadas.

Questões atuais, como a (a) agregação do laudo digital ao procedimento policial, eletrônico ou físico, (b) a identificação biométrica dos investigados, maiores ou adolescentes, (c) a utilização de aplicações que auxiliem na tarefa dos policiais, não só na busca de informações e na facilitação dos trabalhos policiais, mas 
também na organização de ações e procedimentos, (d) a busca de identificação automatizada através de sistemas de reconhecimento facial, (e) a criação de mecanismos de gestão para situações complexas da atualidade, tal qual o meio ambiente, (f) a incorporação de tecnologias embarcadas nas frotas veiculares, (g) a utilização de redes sociais como forma de aproximação comunitária e facilitação da comunicação, e, (h) a disseminação dos conhecimentos através de cursos não presenciais, foram muito bem analisadas pelos nossos autores.

Verifica-se, de outra sorte, que o sistema de Segurança Pública só evolui com a evolução dos seus subsistemas, das polícias militares e civis, das perícias, do sistema prisional, do corpo de bombeiros etc. A comunicação e a tecnologia que a amplia é, de acordo com o idealizador da Teoria dos Sistemas, Niklas Luhmann, o mecanismo de acoplamento estrutural entre os subsistemas e, também, meio de interação com seu entorno, seja com o sistema mãe, a Sociedade, seja com os demais sistemas, o do Direito, o da Política, o da Moral, dentre outros. Certo é que o sistema social acabou por gerar a demanda de um formato de controle dos seres humanos e que a parte criminal primária (de atuação) tenha ficado a cargo das polícias, sobre as quais recai a principal demanda atual: aumento da sensação de segurança.

O ponto de partida da análise sistêmica com base em Luhmann tem por princípio a diferença entre sistemas e seu entorno, sendo aqueles estruturalmente orientados para sua autoorganização, porém não podem existir sem o entorno. Assim, os sistemas se constituem e se mantém mediante a criação e a conservação da diferença com seu entorno, utilizando de seus limites (código e programação) para regular (e preservar) dita diferença. Assim, os sistemas são autopoiéticos autorreferenciais, pois são unidades fechadas e sua organização é tal que o único produto é ele mesmo, evoluindo por seus próprios meios, referem-se a si mesmos e produzem seus elementos constitutivos a partir dos elementos dos quais são compostos. Produzem-se e se reproduzem a si mesmos e suas operações e o modo de reprodução são por natureza autônomos, sendo que a relação que entabulam com o entorno (meio ambiente) é estabelecida (por sua diferenciação funcional) segundo a medida de sua forma operativa. Assim, por serem autopoiéticos, os sistemas são autorreferenciais, auto-organizados e autorreprodutivos. Essa relação - do sistema - estabelecida com o entorno é realizada por meio da comunicação, como referido, que é mecanismo de acoplamento estrutural entre ambos, em estímulos constantes e processamento autoestruturado da informação resultante da diferença sistema/entorno. A comunicação, assim, é o meio pelo qual é possível acesso ao sentido, indispensável para o processo coevolutivo dos sistemas sociais: a comunicação é que faz possível a diferença sistema/entorno.

Aplicando-se essa lógica ao sistema de segurança pública, este sistema não pode existir sem o seu entorno: o indivíduo e, num contexto de estar abrangido, 
a sociedade. O código binário do sistema de segurança pública, por esta teoria, deve estar entre seguro/inseguro ou insegurança/segurança, porém com meandros que vão além dos aspectos objetivos, porquanto a sensação de segurança ou insegurança é algo que pode não ser objetivado e, aliás, amplificado por sistemas comunicacionais e midiáticos.

Assim, o livro, embora não refira diretamente a teoria sistêmica, num contexto de análise de terceira ordem acaba por nos trazer pesquisas que, avaliadas conjuntamente, enfocam aspectos fundamentais do sistema de Segurança Pública e seus aspectos fundamentais diante das tecnologias da informação e da comunicação da contemporaneidade.

Delegado Emerson Wendt Chefe de Polícia na Polícia Civil do Estado do Rio Grande do Sul 



\section{Apresentação}

Este livro é o resultado das pesquisas realizadas por professores e alunos do curso de especialização em Tecnologias da Informação e Comunicação Aplicadas à Segurança Pública e Direitos Humanos (turma 2013-2015), da Universidade Federal de Santa Catarina - Campus Araranguá, financiado pela Secretaria Nacional de Segurança Pública do Ministério da Justiça (Senasp/MJ), com colaboração dos pesquisadores vinculados ao Programa de Pós-Graduação do Mestrado Interdisciplinar em Tecnologias da Informação e Comunicação (PPGTIC/UFSC - Campus Araranguá) e organizado pelo Grupo de Pesquisa do Laboratório de Mídia e Conhecimento (LabMídia/UFSC - Campus Araranguá/CNPq).

As pesquisas aqui apresentadas convergem basicamente para a discussão de três temas fundamentais para a sociedade brasileira: segurança pública, direitos humanos e desenvolvimento das Tecnologias da Informação e Comunicação (TIC).

A convergência destes temas segue a orientação de várias políticas públicas nacionais como ações estratégicas: a consolidação do Sistema Único de Segurança Pública (SUSP), os programas da Secretaria Nacional de Segurança Pública do Ministério da Justiça (Senasp/MJ), o Programa Nacional de Segurança Pública com Cidadania (Pronasci), a criação da Rede Nacional de Altos Estudos em Segurança Pública (Renaesp) e da Matriz Curricular Nacional para Ações Formativas dos Profissionais da Área da Segurança Pública. Da mesma forma, são relevantes à implementação de critérios qualitativos para a distribuição dos recursos do Fundo Nacional de Segurança Pública. No âmbito das políticas públicas de Direitos Humanos, vale ressaltar a terceira edição do Programa Nacional de Direitos Humanos (PNDH-3), o Plano Nacional de Educação em Direitos Humanos (PNEDH) e as várias ações desenvolvidas pela Secretaria de Direitos Humanos da Presidência da República (SDH/PR). Por sua vez, em sua Matriz Curricular Nacional para ações formativas, a Senasp indica como uma das áreas temáticas de capacitação a Comunicação, Informação e Tecnologias em Segurança Pública. 
Essa indicação é corroborada no documento da Agência Brasileira de Desenvolvimento Industrial (ABDI), que coloca como uma das ações mobilizadoras ao desenvolvimento tecnológico e ao crescimento econômico do Brasil no horizonte temporal até 2025 os investimentos estratégicos em TIC em Sistemas de Segurança Pública.

Com estas indicações e a convergência destes três temas - Tecnologias da Informação e Comunicação (TIC), Segurança Pública e Direitos Humanos -, os capítulos a seguir descrevem desde políticas públicas para o uso da TIC, bem como a utilização de tecnologias em áreas específicas da Segurança Pública e a avaliação do próprio curso de pós-graduação em nível de especialização que originou esta obra.

Esperamos que as pesquisas ora expostas possam contribuir com o desenvolvimento de Tecnologias da Informação e Comunicação para Sistemas de Segurança Pública e Direitos Humanos, mas também para a inovação tecnológica direcionada à redução das desigualdades econômicas e à inclusão social.

Boa leitura.

Os organizadores 


\section{Sumário}

CAPÍTULO 1 - POLÍTICAS DE SEGURANÇA PÚBLICA E DIREITOS HUMANOS EM SANTA CATARINA ....................................... 25

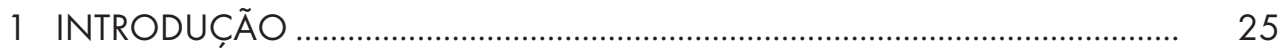

2 PLANOS NACIONAIS DE SEGURANÇA PÚBLICA ......................................... 27

3 POLÍTICAS DE SEGURANÇA PÚBLICA: FHC, LULA E DILMA ……………..... 28

3.1 Secretaria Nacional de Segurança Pública ............................................... 28

3.2 Plano Nacional de Segurança Pública ........................................................ 29

3.3 Sistema Único de Segurança Pública.......................................................... 30

3.4 Programa Nacional de Segurança Pública com Cidadania.................... 31

4 SANTA CATARINA: IMPACTOS DOS PLANOS NACIONAIS -

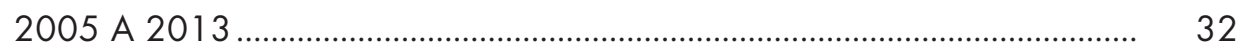

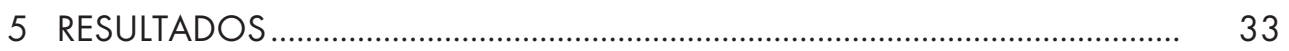

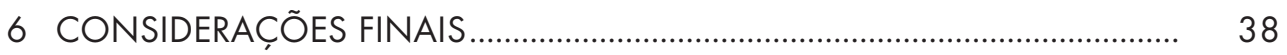

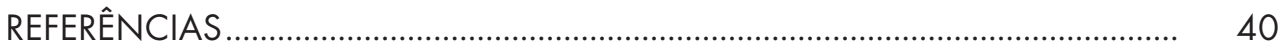

CAPÍTULO 2 - AS TECNOLOGIAS DA INFORMAÇÃO E COMUNICAÇÃO NA GESTÃO ADMINISTRATIVA E OPERACIONAL DA SEGURANÇA PÚBLICA ........................... 43

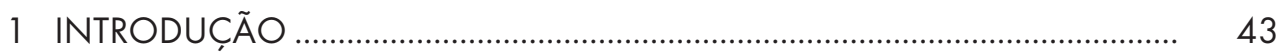

2 PROCEDIMENTOS METODOLÓGICOS ........................................................... 45

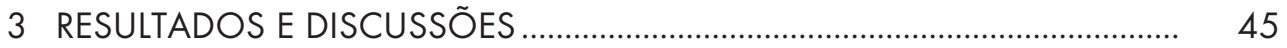


4 CONSIDERAÇÕES FINAIS.

REFERÊNCIAS.

CAPÍTULO 3 - LAUDO DIGITAL - A QUEBRA DE PARADIGMAS .. 55

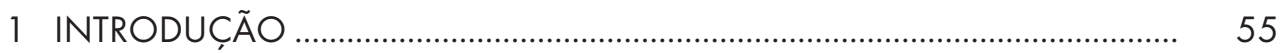

2 PROCEDIMENTOS METODOLÓGICOS …………………………………........ 56

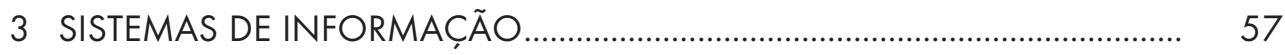

4 FLUXO DE TRABALHO - WORKFLOW …………………………............... 58

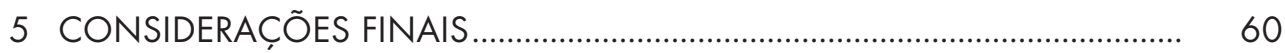

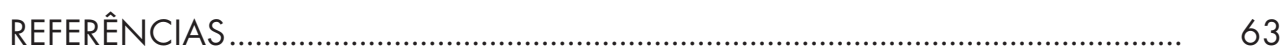

CAPÍTULO 4 - IDENTIFICAÇÃO BIOMÉTRICA DIGITAL NA GESTÃO DO ATENDIMENTO DE ADOLESCENTES INFRATORES ... 65

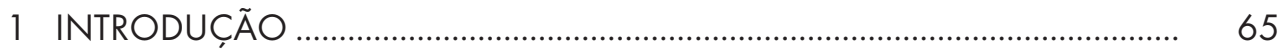

2 CONCEITO E OS TIPOS DE BIOMETRIA ……………................................... 66

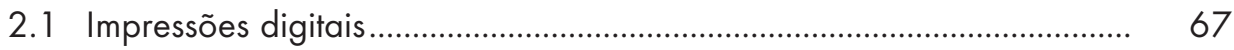

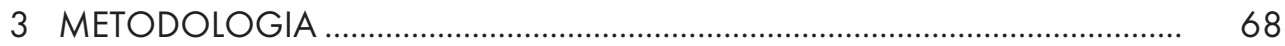

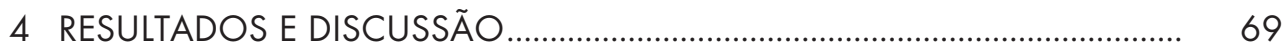

4.1 Comparação dos processos: antes e depois da identificação biométrica ........................................................................... $\quad 72$

4.2 Implantação da identificação biométrica .................................................. 73

5 CONSIDERAÇÕES FINAIS....................................................................

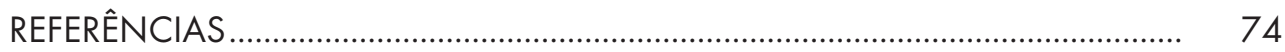

CAPÍTULO 5 - TECNOLOGIAS PARA PREVENÇÃO DE INCÊNDIOS: A TRAGÉDIA DA BOATE KISS

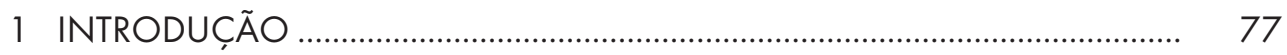

2 ANTECEDENTES: A HISTÓRIA DE UMA TRAGÉDIA ..................................... 78

3 CONCLUSÃO DO INQUÉRITO ………………………………………........ 81 
4 TECNOLOGIAS PARA PREVENÇÃO DE INCÊNDIOS ..................................... 82

4.1 Tecnologias preventivas: sprinkler ……………………………………...... 82

4.2 Sistema de exaustão de fumaça .................................................................... 83

4.3 Detectores de fumaça, térmicos e acionadores manuais.......................... 83

4.4 Sistema de gestão integrado da prevenção de incêndios ........................ 84

5 CONSIDERAÇÕES FINAIS ................................................................................ 89

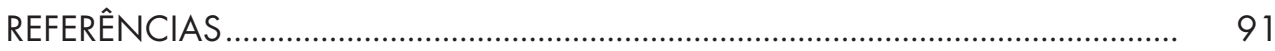

CAPÍTULO 6 - APLICATIVOS PARA DISPOSITIVOS MÓVEIS APLICADOS À SEGURANÇA PÚBLICA .................................. 93

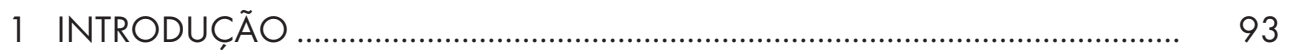

2 DESENVOLVIMENTO ..................................................................................... 95

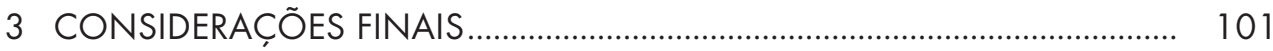

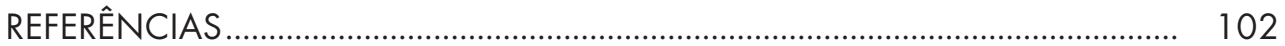

CAPÍTULO 7 - UM MODELO DE DADOS VOLTADO AO SERVIÇO DE INTELIGÊNCIA POLICIAL .......................................... 103

1 INTRODUÇÃO

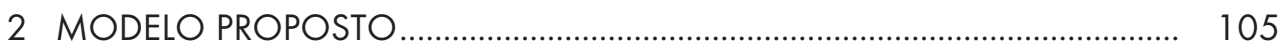

2.1 Modelagem conceitual ........................................................................... 106

3 UTILIZAÇÃO DO MODELO

3.1 Exemplos de consultas e discussões ..................................................... 108

4 CONSIDERAÇÕES FINAIS ............................................................................. 109

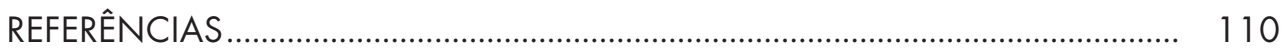

CAPÍTULO 8 - UM ESTUDO SOBRE TÉCNICAS DE BIOMETRIA BASEADAS EM PADRÕES FACIAIS E SUA UTILIZAÇÃO NA SEGURANÇA PÚBLICA

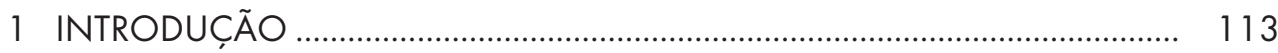

2 SISTEMAS BIOMÉTRICOS ................................................................................ 115 
3 RECONHECIMENTO FACIAL _..................................................................... 116

3.1 Métodos de reconhecimento de rostos ..................................................... 118

3.2 Métodos holísticos ................................................................................ 120

3.3 Métodos estruturais.................................................................................. 121

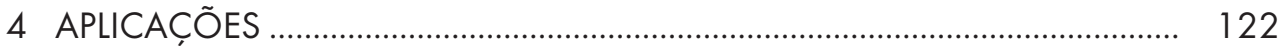

5 USO DO RECONHECIMENTO FACIAL NA SEGURANÇA PÚBLICA .......... 123

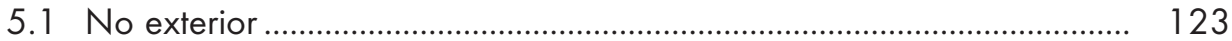

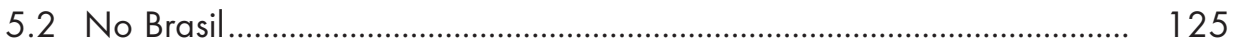

6 CONSIDERAÇÕES FINAIS ................................................................... 128

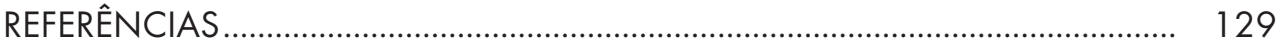

CAPÍTULO 9 - APLICAÇÃO DE IDENTIFICAÇÃO BIOMÉTRICA POR IMPRESSÃO DIGITAL NA LAVRATURA DO AUTO DE PRISÃO EM FLAGRANTE ............................................. 133

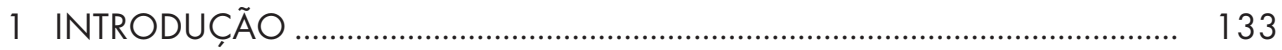

2 AUTO DE PRISÃO EM FLAGRANTE............................................................. 134

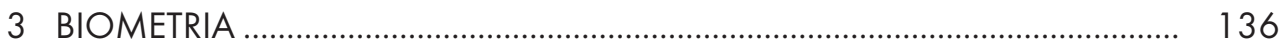

4 CONCEITO E FUNCIONAMENTO ......................................................... 136

5 IMPRESSÃO DIGITAL COMO IDENTIFICADOR BIOMÉTRICO .................... 137

6 SISTEMAS FORENSES DE IDENTIFICAÇÃO DIGITAL.................................... 140

7 EXTRAÇÃO DE CARACTERÍSTICAS, COMO É FEITO NO BRASIL? .............. 141

8 CONCLUSÃO

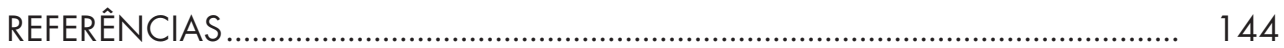

CAPÍTULO 10 - PROPOSTA DE UM SISTEMA DE GESTÃO PARA FISCALIZAÇÃO AMBIENTAL ........................................ 147

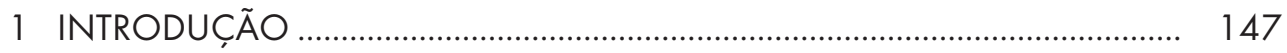

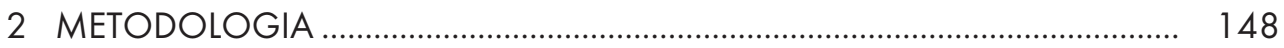


3 FUNDAMENTAÇÃO TEÓRICA ..................................................................... 149

4 GESTÃO AMBIENTAL NA POLÍCIA MILITAR DE MARACAJÁ...................... 150

5 SISTEMA PROPOSTO …………………………..................................... 152

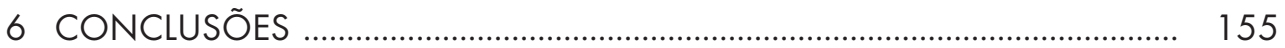

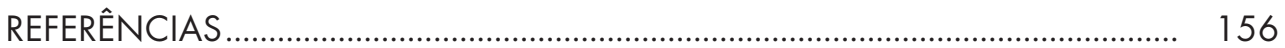

CAPÍTULO 11 - PROPOSTAS PARA INFORMATIZAÇÃO

DE VIATURAS POLICIAIS COMO INSTRUMENTO

DE SEGURANÇA PÚBLICA............................................ 157

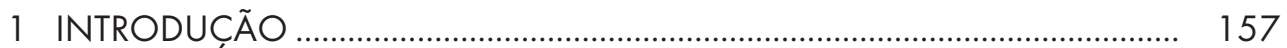

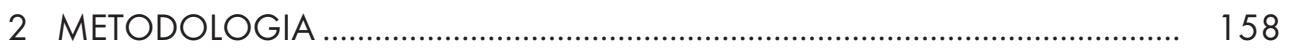

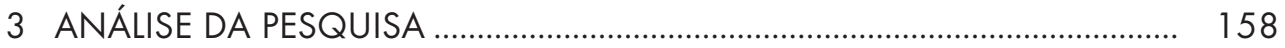

4 PROPOSTA DA VIATURA POLICIAL IDEAL ................................................. 162

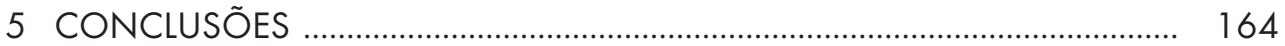

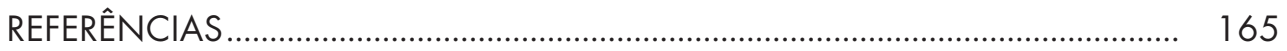

CAPÍTULO 12 - REDES SOCIAIS E A POLÍCIA COMUNITÁRIA: ESTUDO DE CASO DOS CONSEGS DO VALE DO ARARANGUÁ ... 167

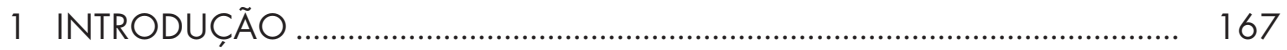

2 OS CONSEGS E AS REDES SOCIAIS ........................................................ 169

3. CONSIDERAÇÕES FINAIS ................................................................... 175

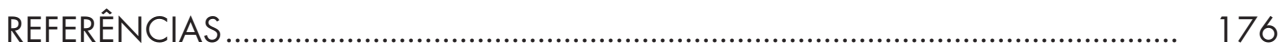

CAPÍTULO 13 - TECNOLOGIAS DA INFORMAÇÃO APLICADAS NA PMSC E BPMA ..................................... 179

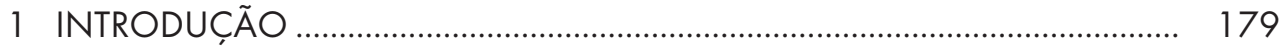

2 PROCEDIMENTOS METODOLÓGICOS .......................................................... 182

3 TIC UTILIZADAS PELA PMSC ...................................................................... 182

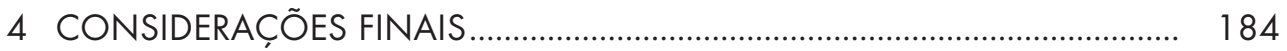

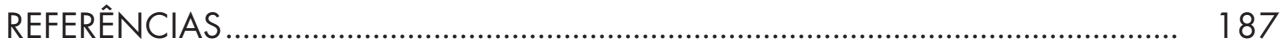


CAPÍTULO 14 - EDUCAÇÃO A DISTÂNCIA, TECNOLOGIA E LEGISLAÇÃO PRISIONAL.................................................. 189

1 INTRODUÇÃO ……………………………………………………………..... 189

2 REFERENCIAL TEÓRICO ……………………………………………...... 190

3 CONSIDERAÇÕES FINAIS ................................................................................ 194

REFERÊNCIAS ............................................................................................ 195

CAPÍTULO 15 - O USO DE TECNOLOGIAS DE INFORMAÇÃO E COMUNICAÇÃO NA MEDIAÇÃO DA ESPECIALIZAÇÃO EM TIC APLICADAS À SEGURANÇA PÚBLICA E DIREITOS HUMANOS ..... 197

1 INTRODUÇÃO …………………………………………………………….... 197

2 FUNDAMENTAÇÃO............................................................................... 198

3 PROCEDIMENTOS METODOLÓGICOS .......................................................... 200

3.1 Coleta de Dados.................................................................................... 200

4 AMBIENTES COMPUTACIONAIS DA UFSC................................................... 201

5 AMBIENTES UTILIZADOS ............................................................................ 201

6 RESULTADO DA PESQUISA APLICADA......................................................... 202

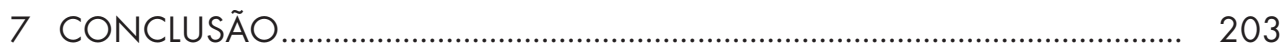

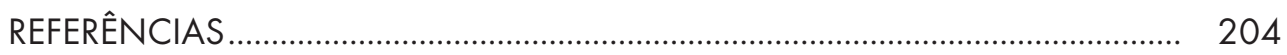

